\title{
Respostas de Componentes Produtivos de Soja a Inoculação de Biológicos em Campo
}

\section{Responses of Soybean Productive Components to Field Biological Inoculation}

\author{
Jorge González Aguilera*a; Alan Mario Zuffo; Rafael Felippe Ratke; Renato Jaqueto Goes ${ }^{\mathrm{b}}$; Natielly Pereira da Silva; \\ Kaline Gabriela de Almeida Marques ${ }^{\mathrm{b}}$; Luane Nathalyne da Silva ${ }^{\mathrm{b}}$; Amanda Camila Silva Trento ${ }^{\mathrm{b}}$; Janaina Tauil Bernardo ${ }^{\mathrm{c}}$ \\ anniversidade Federal do Mato Grosso do Sul, Programa de Pós-Graduação Stricto Sensu em Agronomia. MS, Brasil. \\ bUniversidade Federal do Mato Grosso do Sul. MS, Brasil. \\ 'Universidade Estadual do Rio Grande do Sul. RS, Brasil. \\ *E-mail: j51173@yahoo.com
}

\begin{abstract}
Resumo
O emprego de produtos biológicos no sistema de plantio direto constitui-se uma prática sustentável e com menor potencial poluente que o uso de produtos químicos. Nesta pesquisa objetivou-se avaliar o desempenho agronômico de três cultivares de soja após a inoculação pós emergente de biológicos no solo. O delineamento experimental utilizado foi o de blocos casualizados em esquema em parcelas subdivididas $3 \times 5$, com três repetições. Os tratamentos foram a combinação de três cultivares (BRASMAX-FOCO-IPRO, BRASMAX-DESAFIO-IPRO e BRASMAXBÔNUS-IPRO) e quatro biológicos [Azospirillum brasilense + Bradyrhizobium japonicum (1), BioAx (2), Trichoderma asperrellum (3), $1+2+3$ (4)], mais um Controle (ausência de biológicos) (5). Foi avaliado a altura das plantas (AP), inserção do primeiro legume (IPL), número de legume por planta (PNL), número de grãos por legumes (NGL), massa de mil grãos (MMG) e produtividade (PROD). Os grãos colhidos, foram classificados por tamanho em peneiras de diâmetros de 7.5, 7.0, 6.5, 6.0, 5.5, 5.0 e $4.5 \mathrm{~mm}$. A porcentagem de grãos retidos (RP) e a produtividade relativa de grãos retidos nas peneiras (PRGRP) foi calculada. Os resultados apontaram interações significativas ( $p<0,01)$ para as variáveis NGL, NGP, MMG, Ø6.5, Ø6.0, Ø5.5, Ø5.0, RP e PRGRP. O emprego de biológicos aplicados de modo post-emergente promove uma melhor qualidade fisiológica das sementes de soja se comparadas ao controle, ao considerar os diâmetros de Ø6.0 e Ø6.5.
\end{abstract}

Palavras-chave: Glycine max. Qualidade das Sementes. Azospirillum brasilense; Bradyrhizobium japonicum. BioAx; Trichoderma asperrellum.

\begin{abstract}
The use of biological products in the no-tillage system is a sustainable practice with less polluting potential than the use of chemicals. This research aimed to evaluate the agronomic performance of three soybean cultivars after the post-emergence inoculation of biologicals in the soil. The experimental design used was randomized blocks in a $3 \times 5$ subdivided plots scheme, with three replications. The treatments were the combination of three cultivars (BRASMAX-FOCO-IPRO, BRASMAX-DESAFIO-IPRO and BRASMAX-BÔNUS-IPRO) and four biological [Azospirillum brasilense + Bradyrhizobium japonicum (1), BioAx (2), Trichoderma asperrellum (3), $1+2+3$ (4)], plus a Control (absence of biological products) (5). Plant height (PH), insertion of the first pods (IFP), number of pods per plant (NPP), number of grains per pods $(N G P)$, mass of one thousand grains (MTG) and productivity (PROD) were evaluated. The harvested grains were classified by size in sieves with diameters of 7.5, 7.0, 6.5, 6.0, 5.5, 5.0 and $4.5 \mathrm{~mm}$. The percentage of grains retained (GR) and the relative productivity of grains retained in the sieves (RPGRS) was calculated. The results showed significant interactions $(p<0.01)$ for the variables NGP, NPP, MTG, Ø6.5, Ø6.0, Ø5.5, Ø5.0, GR and RPGRS. The use of biologically applied post-emergence promotes a better physiological quality of soybean seeds compared to the control, when considering the diameters of Ø6.0 and Ø6.5.
\end{abstract}

Keywords: Glycine max. quality of seeds. Azospirillum brasilense. Bradyrhizobium japonicum. BioAx; Trichoderma asperrellum.

\section{Introdução}

A soja (Glycine $\max$ L. Merrill) é uma oleaginosa de grande importância econômica no mercado mundial. Atualmente, pelo seu alto valor proteico é cultivado em todo o mundo e empregada para produzir suplementos alimentícios para animais e o homem, assim como empregada na indústria, para a produção de biocombustíveis (VAL et al., 2014).

A soja é um dos produtos mais importantes para os países exportadores e é um dos alimentos básicos mais importantes dos países que a importam pelo seu elevado valor nutricional. O Brasil é o maior produtor de soja do mundo, com 33\% de toda a produção mundial, depois os Estados Unidos vêm com $32,85 \%$ e, posteriormente, Argentina, com 15,80\% (USDA,
2019). Juntos, esses três países são responsáveis por 79,65\% da safra mundial. No Brasil, a área ocupada pela leguminosa na safra 2018/2019 foi de aproximadamente 36 milhões de hectares e o estado de Mato Grosso do Sul (MS) responde por aproximadamente $8 \%$ da área semeada com soja (CONAB, 2019).

A soja depende de uma boa nutrição do solo para atingir altos níveis de produção, geralmente realizados através do uso de fertilização química, associada a boas práticas de manejo de pragas e doenças. Dentre os manejos atuais, encontrase no Brasil, o manejo em sistemas de plantio direto (SPD) que propicia uma melhor disponibilidade e ciclagem de nutrientes pela decomposição da matéria orgânica, onde os microrganismos formam parte da biota ativa do solo, além de 
ser uma técnica sustentável de cultivo conservacionista em que o plantio é efetuado sem as etapas do preparo convencional da aração e da gradagem (CORREIRA; DURIGAN, 2008).

O emprego de produtos biológicos no SPD constituise uma prática sustentável e com menor potencial poluente que o uso de fertilizantes nitrogenados. A inoculação com bactérias da espécie Bradyrhizobium japonicum podem melhorar os rendimentos da cultura da soja, através da simbiose soja-rizobia como uma importante associação simbiótica. Nesta associação as plantas suprem parte das suas necessidades de nitrogênio $(\mathrm{N})$ por meio da fixação biológica de $\mathrm{N}$, portanto, o uso de fertilizantes nitrogenados pode ser reduzido (POMMERESCHE; HANSEN, 2017). Armendariz et al. (2019) mostraram que a combinação de B. japonicum e Azospirillum brasilense pode ser vantajosa para promover crescimento e produtividade em soja. A. brasilense é uma bactéria de vida livre que, quando inoculada sozinha ou em combinação com B. japonicum, tem demonstrado capacidade de promover a germinação de sementes, a formação de nódulos e o desenvolvimento inicial da soja (CASSÁN et al., 2009).

Na soja, fungos do gênero Trichoderma são comumente empregados como controladores biológicos de fungos patogênicos que acometem a cultura, assim como, tem relatos que alguns isolados podem aumentar o rendimento da cultura (GÕRGEN et al., 2009; MACHADO et al., 2012). Estas características fazem dos fungos do gênero Trichoderma importantes ferramentas de uso no manejo sustentável de cultivos de soja em SPD, por exercerem atividade protetora e estimulante do crescimento das plantas (MACHADO et al., 2012). Alternativamente, a combinação de bactérias fixadoras de $\mathrm{N}$ e fungos que interferem no desenvolvimento de espécies de plantas pode constituir uma ferramenta na recuperação do equilíbrio da biota do solo além de promover um maior rendimento das culturas submetidas a estas combinações.

Assim, o objetivo do trabalho foi determinar o efeito combinado e isolado de biológicos no desempenho de componentes produtivos e o rendimento de peneiras das sementes de três cultivares soja durante a safra 2018/2019 em Chapadão do Sul, MS.

\section{Material e Métodos}

\subsection{Condições experimentais}

O experimento foi conduzido em área experimental da Universidade Federal de Mato Grosso do Sul (UFMS), Campus de Chapadão do Sul, Mato Grosso do Sul (MS) (1846'17,9" de latitude sul, 52³7'25,0" de longitude oeste e altitude média de $810 \mathrm{~m}$ ) na safra 2018/2019. O clima da região está de acordo com a classificação Köppen, do tipo chuvoso tropical (Aw), com verão chuvoso e inverno seco, com precipitação, temperatura média e umidade relativa anual de $1.261 \mathrm{~mm}, 23,97$ ${ }^{\circ} \mathrm{C}$ e $64,23 \%$, respectivamente, o acumulado de precipitação durante a condução do experimento foi de $1.150 \mathrm{~mm}$.

O solo da área experimental é um Latossolo vermelho distrófico com textura argilosa. Antes de iniciar o experimento, amostras de solo foram coletadas a uma profundidade de $0.00-0.20 \mathrm{~m} \mathrm{e}$ as principais propriedades químicas foram determinadas (Quadro 1).

Quadro 1 - Principais propriedades químicas do solo usado no experimento

\begin{tabular}{|c|c|c|c|c|c|c|c|c|c|}
\hline $\mathrm{pH}$ & MO & $\mathrm{P}_{\text {Mehlich-1 }}$ & $\mathrm{H}+\mathrm{Al}$ & $\mathrm{Al}^{3+}$ & $\mathrm{Ca}^{2+}$ & $\mathrm{Mg}^{2+}$ & $\mathrm{K}^{+}$ & CTC & $\mathrm{V}$ \\
\hline $\mathrm{CaCl}_{2}$ & $\mathrm{~g} \mathrm{dm}^{-3}$ & $\mathrm{mg} \mathrm{dm} \mathrm{m}^{-3}$ & \multicolumn{6}{|c|}{ 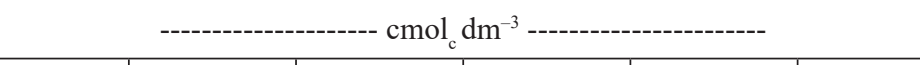 } & $\%$ \\
\hline 4.3 & 22.8 & 12.8 & 5.7 & 0.37 & 2.20 & 0.40 & 0.27 & 8.6 & 33.5 \\
\hline
\end{tabular}

MO: Matéria orgânica. CTC: Capacidade de troca catiônica em pH 7.0. V: Saturação da base.

Fonte: Dados da pesquisa

A correção da acidez do solo foi realizada com a aplicação superficial de 2,5 $\mathrm{t} \mathrm{ha}^{-1}$ de calcário (CaO: 29\%, MgO: 20\%, PRNT: 90,1\%, PN: 101,5\%), com o objetivo de aumentar a saturação por base para $60 \%$. O calcário foi aplicado 60 dias antes da implantação do experimento. A semeadura da soja foi realizada mecanicamente, com auxílio de uma semeadora-adubadora, com distribuição de 13 sementes por metro, com espaçamento entre linhas de $0,45 \mathrm{~m}$. A adubação de base foi constituída por $150 \mathrm{~kg} \mathrm{ha}^{-1}$ de $\mathrm{P}_{2} \mathrm{O}_{5}$, cuja fonte era superfosfato simples. Aos 40 dias após a emergência (DAE), foi realizada a aplicação de adubação foliar dos produtos Actilasa ZM (Zn a $50.22 \mathrm{~g} \mathrm{~L}^{-1}$; S a $41.65 \mathrm{~g} \mathrm{~L}^{-1}$; Mn a $30.01 \mathrm{~g}$ $\mathrm{L}^{-1}$ ) e Racine (Mo a $108.75 \mathrm{~g} \mathrm{~L}^{-1}$; Co a $10.88 \mathrm{~g} \mathrm{~L}^{-1}$; Carbono total $123.25 \mathrm{~g} \mathrm{~L}^{-1}$ ), as doses foram de $1 \mathrm{~L} \mathrm{ha}^{-1}$ e $120 \mathrm{~mL}$ para $\mathrm{ha}^{-1}$, respectivamente.

\subsection{Delineamento experimental}

O delineamento experimental utilizado foi o de parcelas subdivididas de $3 \times 5$, com três repetições. As parcelas foram constituídos por três cultivares de soja [BRASMAX Foco IPRO - hábito de crescimento indeterminado, ciclo médio de 109 dias, grupo de maturação 7.2, BRASMAX Desafio IPRO - hábito de crescimento indeterminado, ciclo médio de 113 dias, grupo de maturação 7.4 e BRASMAX Bônus IPRO - hábito de crescimento indeterminado, ciclo médio de 121 dias, grupo de maturação 7.9] e nas subparcelas cinco biológicos: 1- Azospirillum brasilense (Cepas AbV5 e AbV6) + Bradyrhizobium japonicum (SEMIA 5079 e 5080) ambos na doses de $2 \mathrm{~L} \mathrm{ha}^{-1}$, 2- BioAx® (Citoquininas 3000 ppm, Giberelinas 15 ppm, Auxinas $50 \mathrm{ppm}$ ) na doses de $5 \mathrm{~L} \mathrm{ha}^{-1}$, 3- Trichoderma asperrellum (Isolado SF 04) na doses de 100 
$\mathrm{g} \mathrm{ha}^{-1}$, 4- a combinação dos três biológicos empregando as mesmas doses deles isoladamente, e 5- ausência de biológicos. Os biológicos foram aplicados três dias após o plantio empregando bomba costal de $20 \mathrm{~L}$ nas doses recomendadas por tratamento e fazendo a diluição dos produtos comerciais em água.

As sementes foram semeadas sem nenhum tratamento de sementes, afim de garantir o efeito isolado dos biológicos aplicados após o plantio numa área com histórico de manejo em SPD. Cada unidade experimental foi constituída de 6 linhas de $3 \mathrm{~m}$ de comprimento, sendo as duas linhas laterais de cada lado desconsideradas e, 0,50 m em cada extremidade, constituindo assim a área útil utilizada em todas as análises de nosso experimento de 2 linhas de $2 \mathrm{~m}$ cada. Durante todo o ciclo da cultura, o controle de plantas daninhas, pragas e doenças nocivas foi realizado com produtos químicos de acordo com a necessidade.

\subsection{Variáveis mesuradas no experimento}

No estágio da maturação da soja realizou-se a colheita da área útil de cada parcela, e dentro dela cinco plantas foram selecionadas por parcela e nelas foram avaliadas as seguintes variáveis: altura das plantas (AP) $(\mathrm{cm})$, determinada pela medida da distância da superfície do solo até a inserção da última folha com a ajuda de uma régua milimétrica; inserção da primeira legume (IPL) $(\mathrm{cm})$, determinada pela medição da distância da superfície do solo até a inserção da primeira legume com o auxílio de uma régua milimétrica; número de legumes por planta (NLP) (unidade), determinado pela contagem do número total de legumes nas cinco plantas e pela divisão desse valor pelo número de plantas; número de grãos por legumes (NGL) (unidade), determinado pela contagem manual de todos os grãos divididos pela NLP; massa de mil grãos (MMG) (g), determinada de acordo com a metodologia descrita no Brasil (2009); produtividade dos grãos (PROD) $\left(\mathrm{kg} \mathrm{ha}^{-1}\right)$, realizada pela colheita de todas as plantas na área útil $\left(1,8 \mathrm{~m}^{2}\right)$ e padronizando o peso para $13 \%$ de umidade dos grãos. Para avaliar o tamanho das sementes, três amostras de $100 \mathrm{~g}$ de grãos foram peneiradas e o peso retido em cada peneira foi quantificado para as peneiras com diâmetros de 7,5 $\mathrm{mm}$, 7,0 mm, 6,5 mm, 6,0 mm, 5,5 mm, 5,0 mm e 4,5 mm.

Para obter uma classificação de cada parcela para posterior comparação, a massa dos grãos retidos em cada peneira foi atribuída um peso (notas), na qual, para a peneira $\varnothing 7,5$, foi estipulado o peso 2; para peneira $\varnothing 7.0$, peso 6; para peneira $\varnothing 6,5$, peso 10 ; para peneira $\varnothing 6.0$, peso 10 ; para peneira $\varnothing 5,5$, peso 6; para a peneira $\varnothing 5.0$, peso 4 , e para a peneira $\varnothing 4.5$, peso 1 (16). Essas medidas de peso/nota foram estabelecidas devido ao requisito referente ao padrão exigido no tamanho das sementes no mercado de sementes de soja. Por meio dos resultados coletados, foi desenvolvida a seguinte fórmula descrita por Carbonell et al. (2010) com modificações:
$\mathrm{PRGRT}=(\mathrm{P} 7,5 \times \mathrm{Peso} 2+\mathrm{P} 7,0 \times \mathrm{Peso} 6+\mathrm{P} 6,5 \times \mathrm{Peso} 10+\mathrm{P} 6,0 \times \mathrm{Pe}-$ so10+P5,5xPeso6+P5,0xPeso4+P4,5xPeso1)/(P7,5+P7,0+P6 $, 5+\mathrm{P} 6,0+\mathrm{P} 5,5+\mathrm{P} 5,0+\mathrm{P} 4,5)$

Onde PRGRT: produtividade relativa de grãos retidos nas peneiras; P7.5: massa $(\mathrm{g})$ retida na peneira de furo redondo do número 07.5 ; P7.0: massa $(\mathrm{g})$ retida na peneira de furo redondo do número Ø7.0; P6.5: massa (g) retida na peneira de furo redondo do número Ø6.5; P6.0: massa (g) retida na peneira de furo redondo do número Ø6.0; P5.5: massa (g) retida na peneira de furo redondo do número Ø5.5; P5.0: massa $(\mathrm{g})$ retida na peneira de furo redondo do número Ø5.0 e P4.5: massa ( $\mathrm{g}$ ) retida na peneira de furo redondo do número $\varnothing 4.5$.

Também foi determinado o rendimento de peneira (RP \%), conforme a fórmula:

\section{$\mathrm{RP}=(\mathrm{P} 70+\mathrm{P} 65+\mathrm{P} 60+\mathrm{P} 55) /(\mathrm{P} 75+\mathrm{P} 70+\mathrm{P} 65+\mathrm{P} 60+\mathrm{P} 55+\mathrm{P} 50+$ P45+Fundo)}

em que RP \%: rendimento de peneira; P75: massa (g) retida na peneira de furo redondo de número 7.5; P70: massa (g) retida na peneira de furo redondo de número 7.0; P65: massa $(\mathrm{g})$ retida na peneira de furo redondo de número 6.5 ; P60: massa $(\mathrm{g})$ retida na peneira de furo redondo de número 6.0; P55: massa (g) retida na peneira de furo redondo de número 5.5; P50: massa $(\mathrm{g})$ retida na peneira de furo redondo de número 5.0; e P45: massa (g) retida na peneira de furo redondo de número 4.5 (CARBONELL et al., 2010).

\subsection{Análises estatísticas}

Os dados experimentais foram submetidos aos testes de verificação das premissas de normalidade e homogeneidade de variância. Os dados foram submetidos à análise de variância (ANOVA) e, quando significativas, as médias foram comparadas pelo teste de 5\% de Scott-Knott, utilizando o programa estatístico Rbio (BHERING, 2017).

\section{Resultados e Discussão}

Resultados da análise de variância contendo os efeitos de cultivares (C) e biológicos (B) sob os componentes de rendimento, produção e qualidade de grãos de soja são mostrados no Quadro 1. Efeitos significativos para a interação CxB foram encontrados para as variáveis NGL, NGP, MMG, PRGRP, Ø6.5, Ø6.0, Ø5.5, Ø5.0 e RP (dados não mostrados). Os efeitos dos fatores individuais para cultivares nas variáveis AP, IPL, NLP, PROD e $\varnothing 7.0$ (Figura 1) e para biológicos na variável NLP (Figura 2) são apresentados.

Na Figura 1, o efeito das cultivares sob três componente de produção (AP, IPL e NLP), a PROD e a peneira $\varnothing 7.0$ é mostrada. Para as variáveis AP e IPL, as cultivares se diferenciaram de igual forma, com valores que foram superiores para BRASMAX Bônus IPRO > BRASMAX Foco IPRO > BRASMAX Desafio IPRO, com diferencias que foram significativas pelo teste Scott-Knott $(p<0.05)$. 
Estas três cultivares empregadas no experimento formam parte das cultivares mais cultivadas no cerrado brasileiro. Elas fenotipicamente são consideradas de ciclo médio a precoce, porém, tem vários atributos que as diferencia e ao mesmo tempo as torna excelentes opções de cultivos para os produtores nas diferentes janelas de semeadura da região. Quando considerado o NLP, a diferenciação entre as cultivares foi distinta sendo a BRASMAX Desafio IPRO a de maior valor com 55 legumes por planta, diferenciando-se das outras duas cultivares que entre elas não se diferenciaram pelo teste Scott-Knot $(p<0.05)$. Estas variações estão relacionadas as diferenças no potencial genético, ciclo e outras características intrínsecas de cada cultivar (FELISBERTO et al., 2015; SOARES et al., 2015).

A PROD (Figura 1), é umas das principais características que melhoristas e produtores almejam sempre no sentido positivo (aumento do rendimento), sendo para esta variável a maior expressão dos rendimentos obtidos nas cultivares BRASMAX Desafio IPRO e BRASMAX Bônus IPRO com 4868 e $4856 \mathrm{~kg} \mathrm{ha}^{-1}$ respectivamente, próximos das médias nacionais para a região que foram de 6168 e $5580 \mathrm{~kg} \mathrm{ha}^{-1}$ respectivamente, na mesma safra 2018/2019 (BRASMAX, 2019). Para a cultivar BRASMAX Foco IPRO verificou-se produtividade de $4135 \mathrm{~kg} \mathrm{ha}^{-1}$, valores próximos aos obtidos Brasmax (2019), que foram de $5280 \mathrm{~kg} \mathrm{ha}^{-1}$ na região.

A peneira de $\varnothing 7.0$ dentre as peneiras avaliadas foi $o$ segundo maior tamanho aferido, e com ele o maior diâmetro dos grãos retidos nela visto que na peneira 07.5 não se manifestaram valores em nenhuma das três cultivares testadas (Figura 1). Ao comparar as três cultivares, verificou-se que a cultivar BRASMAX Bônus IPRO foi a que apresentou a maior retenção de grão para esta peneira com $35 \%$ dos grãos, muito acima do retido pela BRASMAX Foco IPRO e BRASMAX Desafio IPRO que obtiveram 0.28 e $4.48 \%$, respectivamente. $\mathrm{O}$ tamanho do grão está muito associado ao desempenho genético e fenotípico do cultivar o que determina esse tamanho do grão nas diferentes cultivares. Embora, que poucos estudos quantificam essa distribuição nas diferentes peneiras, característica muito descrita para feijão (Phaseolus vulgaris L.), para soja, a demanda principal neste sentido é a uniformidade do tamanho no grão para ter uma melhor distribuição e uniformidade no plantio, partindo no nível tecnológico alcançado na semeadura atual no Brasil, e de tal modo, obter uma densidade de semeadura adequada. Pádua et al. (2010) verificaram que sementes menores (peneira 4,0 $\mathrm{mm}$ ) produzem plantas com menor altura na colheita e menor produtividade, em relação às sementes maiores (peneira 6,0 $\mathrm{mm}$ ); já as sementes maiores (peneira 7,0 mm) apresentam maiores porcentagens de germinação e de vigor. Biruel, Paula e Aguiar (2010) relataram que existe uma relação direta entre o tamanho da semente e o vigor e germinação, associado com a qualidade fisiológica, onde as sementes pequenas apresentam menores valores de germinação e vigor que as de tamanho médio e grande quando comparadas.
Figura 1 - Comparação de médias obtida em componentes de produção de três cultivares de soja avaliados em experimento com aplicação de biológicos conduzidos em campo na safra de 2018/2019, Chapadão do Sul -MS. Letras diferentes significam diferenças estatísticas ao $p<0.05$ pelo Teste Scott - Knott. ( $\mathrm{n}=$ 60)
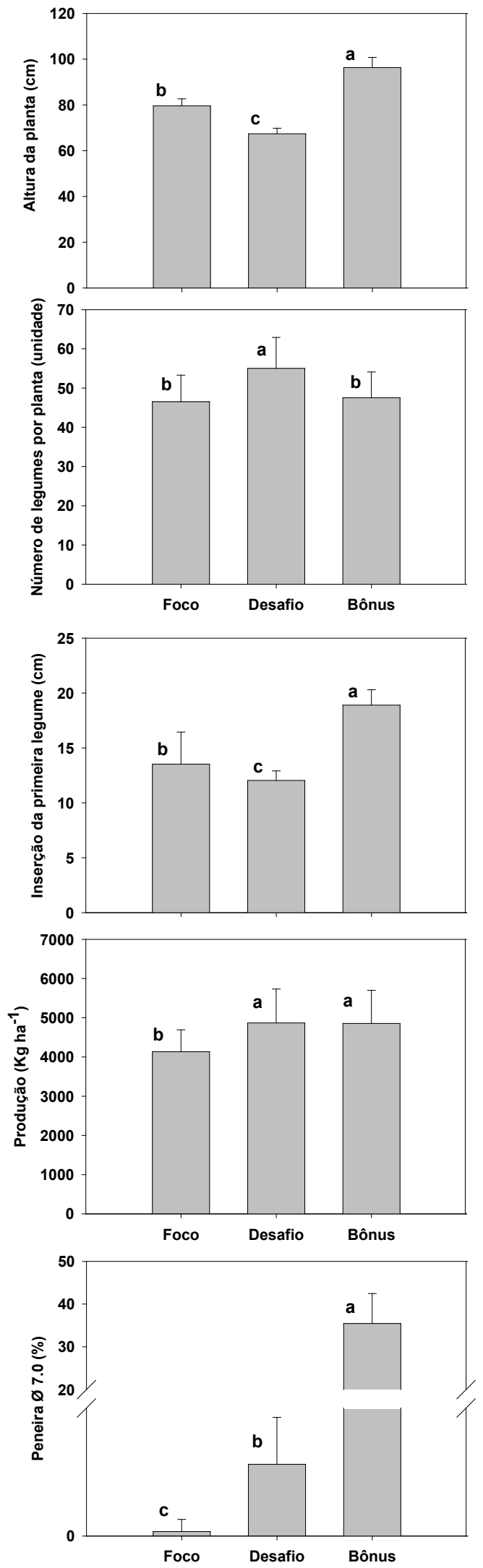

Fonte: Dados da pesquisa. 
Na Figura 2, o efeito da co-inoculação com biológicos sob o componente de produção NLP é mostrada. Para a variável representada na Figura 2, houve efeito isolado dos biológicos em relação ao controle (ausência de biológicos), sendo a combinação dos três biológicos empregados igual ao controle estatisticamente, com valores de 51 e 53 respectivamente, ao mesmo tempo que eles foram superiores ao emprego dos biológicos de modo isolado para esta variável. O sinergismo dos biológicos pode ter contribuído para esta resposta obtida, reportada já em outros trabalhos (FRANCO et al., 2015; KHAN et al., 2017).

Figura 2 - Comparação de médias obtida em componentes de produção de três cultivares de soja avaliados em experimento com aplicação de biológicos conduzidos em campo na safra de 2018/2019, Chapadão do Sul -MS. (1): Azospirillum brasilense e Bradyrhizobium japonicum, (2): BioAx, (3): Trichoderma asperrellum, Controle: ausência de biológicos. Letras diferentes significam diferenças estatísticas ao $p<0.05$ pelo Teste Scott Knott. $(\mathrm{n}=36)$.

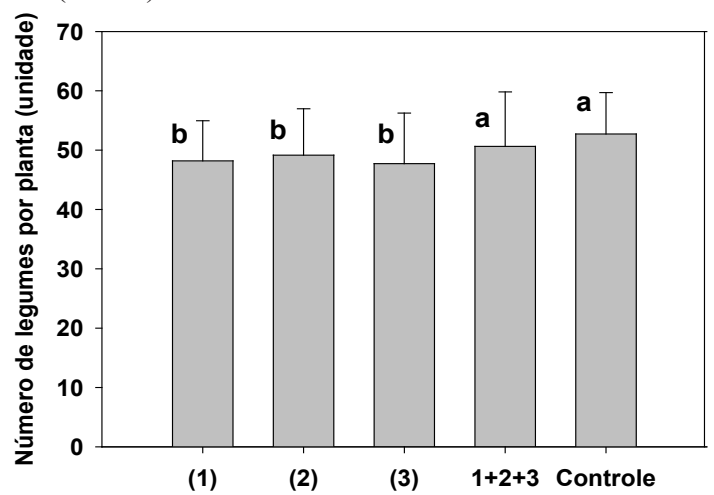

Fonte: Dados da pesquisa.
A coinoculação com biológicos nas três cultivares de soja empregadas quando consideradas as variáveis NGL, NGP, MMG, as peneiras Ø6.5, Ø6.0, Ø5.5, Ø5.5, RP e PRGRP evidenciou interações $\mathrm{CxB}$ para todas elas, e as comparações pelo teste Scott-Knot $(p<0.05)$ são mostradas no Quadro 2. A coinoculação com $A$. brasilense e $B$. japonicum tem sido relatada com efeitos benéficos sob a cultura da soja se consideramos os relatos de Rego et al. (2018), Alberto-Casas et al. (2019) e Armendariz et al. (2019), entretanto, outros autores como Zuffo et al. (2015) não obtiveram efeitos positivos com a sua aplicação ao avaliar também a coinoculação em soja. Nas condições atuais do Brasil, a coinoculação com estas duas bactérias como tratamento de sementes é quase uma pratica comum para a maioria dos produtores. Quando considerado isoladamente esta combinação (A. brasilense e $B$. japonicum) as cultivares se diferenciaram para todas as variáveis sendo a resposta variável como observado no Quadro 2, com a exceção da RP que não se obteve diferenças significativas dentre as cultivares para este tratamento. Para o NGL, Ø6.0, Ø5.0 e PRGRP as cultivares BRASMAX Foco IPRO (2.51 grãos legume ${ }^{-1}, 55.89 \%, 3.09 \%$ e 8.66 respectivamente) e BRASMAX Desafio IPRO (2.50 grãos legume ${ }^{-1}$, 50.37\%, $2.22 \%$ e 8.93 respectivamente) foram as melhores, por sua vez, para NGP a BRASMAX Desafio IPRO (134.73 grãos planta $^{-1}$ ) foi superior as outras cultivares, na MMG e a peneira Ø6.5 a BRASMAX Bônus IPRO foi a superior com $198.13 \mathrm{~g}$ e $41.32 \%$ respectivamente, e na peneira $\varnothing 5.5$ a BRASMAX Foco IPRO foi a superior com $26.22 \%$ de grãos retidos quando comparados pelo teste Scott-Knot $(p<0.05)$.

Quadro 2 - Comparação de médias em variáveis com interação associadas aos componentes de produção de soja obtidos em experimento com aplicação post-emergente de biológicos conduzidos em campo na safra de 2018/2019, Chapadão do Sul - MS

\begin{tabular}{|c|c|c|c|c|c|c|c|c|c|}
\hline \multirow{2}{*}{ Biológicos } & \multicolumn{2}{|c|}{$\mathrm{NGL}^{1}\left(\right.$ grãos legume $\left.^{-1}\right)$} & \multicolumn{3}{|c|}{ NGP $\left(\right.$ grãos planta $\left.^{-1}\right)$} & \multicolumn{3}{|c|}{ MMG $(g)$} & \multirow[b]{2}{*}{ Bônus } \\
\hline & Foco & Desafio & Bônus & Foco & Desafio & Bônus & Foco & Desafio & \\
\hline $\mathrm{AB}(1)$ & $2,51 \mathrm{aB}$ & $2,50 \mathrm{aA}$ & $1,96 \mathrm{bA}$ & $108,6 \mathrm{bA}$ & $134,73 \mathrm{aA}$ & $92,12 \mathrm{cA}$ & $155,94 \mathrm{bA}$ & $151,91 \mathrm{bB}$ & $198,13 \mathrm{aA}$ \\
\hline Bio-Ax (2) & $2,48 \mathrm{aB}$ & $2,47 \mathrm{aA}$ & $1,96 \mathrm{bA}$ & $122,25 \mathrm{aA}$ & $130,82 \mathrm{aA}$ & $88,53 \mathrm{bA}$ & $144,29 \mathrm{cB}$ & $163,64 \mathrm{bA}$ & $199,97 \mathrm{aA}$ \\
\hline Trich. (3) & $2,73 \mathrm{aA}$ & $2,50 \mathrm{bA}$ & $1,99 \mathrm{cA}$ & $115,95 \mathrm{bA}$ & $135,42 \mathrm{aA}$ & $89,52 \mathrm{cA}$ & $139,22 \mathrm{bB}$ & $149,43 \mathrm{bB}$ & $200,61 \mathrm{aA}$ \\
\hline $1+2+3$ & $2,34 \mathrm{aC}$ & $2,49 \mathrm{aA}$ & $1,98 \mathrm{bA}$ & $114,25 \mathrm{bA}$ & $139,03 \mathrm{aA}$ & $93,63 \mathrm{cA}$ & $137,14 \mathrm{cB}$ & $148,29 \mathrm{bB}$ & $195,87 \mathrm{aA}$ \\
\hline \multirow[t]{2}{*}{ Controle } & $2,27 \mathrm{aC}$ & $2,44 \mathrm{aA}$ & $1,98 \mathrm{bA}$ & $105,07 \mathrm{bA}$ & $143,20 \mathrm{aA}$ & $105,40 \mathrm{bA}$ & $144,28 \mathrm{bB}$ & $144,65 \mathrm{bB}$ & $183,47 \mathrm{aB}$ \\
\hline & \multicolumn{2}{|c|}{$\varnothing 6.5(\%)$} & \multicolumn{3}{|c|}{$\varnothing 6.0(\%)$} & \multicolumn{3}{|c|}{$\varnothing 5.5(\%)$} & \\
\hline $\mathrm{AB}(1)$ & $12,07 \mathrm{cA}$ & $24,67 \mathrm{bA}$ & $41,32 \mathrm{aA}$ & & $50,37 \mathrm{aA}$ & $15,83 \mathrm{bA}$ & $26,22 \mathrm{aE}$ & $16,77 \mathrm{bB}$ & $2,94 \mathrm{cA}$ \\
\hline Bio-Ax (2) & $8,63 \mathrm{cB}$ & $22,98 \mathrm{bA}$ & $41,58 \mathrm{aA}$ & $53,97 \mathrm{aA}$ & $50,90 \mathrm{aA}$ & $28,27 \mathrm{bB}$ & $32,18 \mathrm{aD}$ & $34 \mathrm{bB}$ & $3,42 \mathrm{cA}$ \\
\hline Trich. (3) & $7,00 \mathrm{cC}$ & $23,04 \mathrm{bA}$ & $41,29 \mathrm{aA}$ & $51,47 \mathrm{aA}$ & $50,49 \mathrm{aA}$ & $15,60 \mathrm{bA}$ & $36,25 \mathrm{aC}$ & $20,17 \mathrm{bB}$ & $2,54 \mathrm{cA}$ \\
\hline $1+2+3$ & $5,35 \mathrm{cC}$ & $19,31 \mathrm{bB}$ & $44,45 \mathrm{aA}$ & $48,29 \mathrm{aA}$ & $48,40 \mathrm{aA}$ & $16,88 \mathrm{aB}$ & $39,12 \mathrm{aB}$ & $22,70 \mathrm{bA}$ & $2,72 \mathrm{cA}$ \\
\hline \multirow[t]{2}{*}{ Controle } & $2,96 \mathrm{cD}$ & $23,58 \mathrm{bA}$ & $37,91 \mathrm{aB}$ & $35,55 \mathrm{bB}$ & $46,23 \mathrm{aA}$ & $16,24 \mathrm{aB}$ & $48,06 \mathrm{aA}$ & $\mathrm{bB}$ & $3,32 \mathrm{cA}$ \\
\hline & \multicolumn{2}{|c|}{$\varnothing 5.0(\%)$} & \multicolumn{3}{|c|}{$\mathrm{RP}(\%)$} & \multicolumn{3}{|c|}{ PRGRP (uni) } & \\
\hline $\mathrm{AB}(1)$ & $3.09 \mathrm{aD}$ & $2.22 \mathrm{aB}$ & $0.43 \mathrm{bB}$ & $96.05 \mathrm{aA}$ & $97.38 \mathrm{aA}$ & $96.88 \mathrm{aA}$ & $8,66 \mathrm{aA}$ & $8,93 \mathrm{aA}$ & $8,18 \mathrm{bA}$ \\
\hline Bio-Ax (2) & $3.88 \mathrm{aD}$ & $2.59 \mathrm{aB}$ & $0,62 \mathrm{bB}$ & $95.26 \mathrm{aA}$ & $97.01 \mathrm{aA}$ & $96.42 \mathrm{aA}$ & $8,39 \mathrm{bB}$ & $8,89 \mathrm{aA}$ & $8,30 \mathrm{bA}$ \\
\hline Trich. (3) & $4.97 \mathrm{aC}$ & $2.75 \mathrm{bB}$ & $0.24 \mathrm{cB}$ & $94.24 \mathrm{bA}$ & $96.81 \mathrm{aA}$ & $96.81 \mathrm{aA}$ & $8,18 \mathrm{bC}$ & $8,86 \mathrm{aA}$ & $8,16 \mathrm{bA}$ \\
\hline $1+2+3$ & $6.24 \mathrm{aB}$ & $4.34 \mathrm{bA}$ & $0.32 \mathrm{cB}$ & $92.03 \mathrm{cB}$ & $94.87 \mathrm{bB}$ & $97.70 \mathrm{aA}$ & $7,92 \mathrm{bD}$ & $8,59 \mathrm{aB}$ & $8,34 \mathrm{aA}$ \\
\hline Controle & $10.89 \mathrm{aA}$ & $4.32 \mathrm{bA}$ & $3,04 \mathrm{bA}$ & $87.23 \mathrm{cC}$ & $94.10 \mathrm{aB}$ & $90.79 \mathrm{bB}$ & $7,24 \mathrm{cE}$ & $8,70 \mathrm{aB}$ & $7,81 \mathrm{bB}$ \\
\hline
\end{tabular}

Letras diferentes maiúsculas na coluna e minúsculas na linha significam diferenças significativas ao $p<0.05$ pelo Teste Scott-Knott. NGL: número de grãos por legume (uni); NGP: número de grão por planta (uni); MMG: massa de mil grãos (g); PROD: produção (kg); Ø7.0: percentagem de grãos retidos na peneira $7 \mathrm{~mm}$; Ø6.5: percentagem de grãos retidos na peneira $6.5 \mathrm{~mm}$; Ø6.0: percentagem de grãos retidos na peneira $6 \mathrm{~mm}$; Ø5.5: percentagem de grãos retidos na peneira $5.5 \mathrm{~mm}$; Ø5.0: percentagem de grãos retidos na peneira $5 \mathrm{~mm}$; RP: rendimento de peneiras (\%). PRGRP: produtividade relativa de grãos retidos na peneira (uni); CV: coeficiente de variação (\%). (1): Azospirillum brasilense + Bradyrhizobium japonicum, (2): BioAx, (3): Trichoderma asperrellum, Controle: ausência de biológicos.

Fonte: Dados da pesquisa. 
Estes resultados evidenciam a complexidade destas interações obtidas mostrando que elas foram sempre dependentes das cultivares obtidas.

O Bio-Ax é uma mistura de bactérias que é recomendado para promover na planta uma melhor assimilação de nutrientes segundo os obtentores desse produto (AMK, 2019), entretanto, nenhum trabalho tem sido publicado mostrando esses benefícios. O comportamento das três cultivares testadas com este produto biológico demonstrou diferenças significativas $(p<0.05)$ para todas as variáveis apresentadas no Quadro 2, com a exceção da variável RP aonde as cultivares não se diferenciaram. As respostas das cultivares variam, com destaque para as cultivares BRASMAX Foco IPRO e BRASMAX Desafio IPRO em relação a BRASMAX Bônus IPRO nas variáveis NGL, NGP, peneira Ø6.0 e Ø5.0. A cultivar BRASMAX Bônus IPRO se destacou quando observado o comportamento da variável MMG e Ø6.5, proporcionando assim os maiores pesos de mil grãos com $199.97 \mathrm{~g}$ e as maiores percentagens de grãos na peneira Ø6.5 com $41.58 \%$ dos grãos retidos nessa peneira, mostrando o potencial que esta cultivar teve em relação às outras quando aplicado o Bio-Ax, resultados que foram superiores ao da testemunha (Quadro 2).

Fungos do gênero Trichoderma spp. têm sido empregados de modo frequente no controle de doenças que acometem as raízes de várias espécies em condições de campo (BETTIOL; MORANDI, 2009; MACHADO et al., 2012; BERNARDO et al., 2019), assim como, existem relatos que mostram o efeito deles na promoção do crescimento e produtividade das plantas, resistência a doenças e a estres abiótico (GÕRGEN et al., 2009; MASTOURI; BJÖRKMAN; HARMAN, 2010; JUNGES et al., 2016). Estas vantagens são observadas ao aplicar T. asperrellum em campo, obtendo-se uma resposta que foi dependente do cultivar empregada, diferenciandose elas em todas as variáveis apresentadas no Quadro 2. A cultivar BRASMAX Foco IPRO mostrou-se superior quando avaliada a NGL e as peneiras Ø5.5 e Ø5.0, comportamento que ressalta que ainda que produz o maior número de grãos por legumes apresentou eles na maior percentagem nas peneiras de menor diâmetro. A cultivar BRASMAX Desafio IPRO foi destaque no NGP, RP e PRGRP com 135,42 grãos planta $^{-1}$, $96.81 \%$ e 8,86 respectivamente, manifestando assim que para este tratamento com T. asperrellum em campo a cultivar consegue além de produzir o maior número de grãos, $96.81 \%$ deles estavam dentre os tamanhos de peneiras de maior peso segundo o critério empregado em nosso trabalho.

A cultivar BRASMAX Bonûs IPRO pela sua vez se destacou nas variáveis MMG e na peneira Ø6.5, evidenciando assim uma maior massa dos grãos em combinação com um tamanho maior se consideramos que $41.29 \%$ dos grãos foram retidos na peneira Ø6.5 (Quadro 2). Estas evidências demostram os efeitos positivos que se obtém ao aplicar $T$. asperrellum em campo na cultura da soja que corrobora os achados em outras pesquisas empregando fungos do mesmo gênero (GÖRGEN et al., 2009; MASTOURI; BJÖRKMAN; HARMAN, 2010; JUNGES et al., 2016). A resposta da combinação dos biológicos $(1+2+3)$ também foi avaliada como um tratamento, e como os biológicos de modo individual, a resposta obtida foi dependente das cultivares empregadas, não existindo um padrão nessa resposta obtida (Quadro 2). Na variável NGL, a resposta obtida foi igual a obtida ao aplicar de modo individual AB e Bio-Ax, assim como no Controle, manifestando as maiores médias para as cultivares BRASMAX Foco IPRO e BRASMAX Desafio IPRO com 2.34 e 2.49 grãos legume , $^{-1}$, respectivamente.

Para as variáveis NGP, Ø6.5 e Ø5.0 o comportamento foi similar ao obtido com $T$. asperrellum apenas, sendo a cultivar BRASMAX Desafio IPRO superior ao restante das cultivares com 139.03 grãos planta ${ }^{-1}, 44.45 \%$ e $6.24 \%$ respectivamente. A MMG manifestou resposta similar a obtida pelo tratamento com Bio-Ax para esta variável com 195.87 g para a cultivar BRASMAX Bônus IPRO. Para a peneira Ø6.0 as cultivares não se diferenciaram para este tratamento pelo teste ScottKnot $(p<0.05)$, já a peneira Ø5.5 manteve o mesmo comportamento de todos os biológicos aplicados de modo individualmente e até o controle, sendo a cultivar BRASMAX Foco IPRO inferior as outras duas cultivares com 39.12\% dos grãos retidos nessa peneira, si considera-os o menor tamanho desta peneira. A combinação de biológicos promoveu o melhor desenvolvimento da soja se considerar-nos que para a maioria das variáveis associadas aos componentes produtivos e de qualidade ele teve destaque em relação ao controle.

Ao comparar entre os biológicos em relação ao controle, de modo geral os biológicos se manifestaram na maioria das vezes superiores (Quadro 2). Para a variável NGL no cultivar BRASMAX Foco IPRO o tratamento Trich. foi o superior quando comparado com os demais tratamentos. Entretanto, para as outras duas cultivares empregadas não se observaram estas diferenças, ainda que este tratamento Trich. continuou a ter as maiores médias. O NGP por sua vez não foi influenciado pelos biológicos observando-se que as médias não se diferenciaram em relação ao controle. Os biológicos quando observada a MMG manifestaram diferenças estatísticas pelo teste Scott-Knot $(p<0.05)$, sendo superiores os tratamentos com AB, Bio-Ax e a combinação de todos os biológicos em relação ao controle nas cultivares BRASMAX Foco IPRO, BRASMAX Desafio IPRO e BRASMAX Bônus IPRO respectivamente. Ao considerar as peneiras procura-se que os tratamentos contribuíam com a maior percentagem de grãos nas peneiras mais graúdas e nas menores essa percentagem seja menor, porém muitas vezes essa variação depende do cultivar empregada se considerarmos que a cultivar BRASMAX Foco IPRO, BRASMAX Desafio IPRO e BRASMAX Bônus IPRO manifestaram as maiores percentagens nas peneiras $\varnothing 6.0$, $\varnothing 6.0$ e $\varnothing 6.5$ respectivamente, com valores próximos do $50 \%$ dos grãos retidos nessas peneiras. Na peneira $\varnothing 6.5$ no cultivar BRASMAX Foco IPRO o tratamento que mais estimulou essa retenção de peneira foi o $\mathrm{AB}$ diferenciando-se do 
restante dos tratamentos, já na BRASMAX Desafio IPRO está diferenciação foi menor se consideramos que os tratamentos superiores foram todos os biológicos e o controle, entretanto, na cultivar BRASMAX Bônus IPRO todos os biológicos e sua combinação foram superiores ao controle. Na peneira Ø6.0 no cultivar BRASMAX Foco IPRO todos os biológicos foram superiores ao controle, já na cultivar BRASMAX Desafio IPRO não houve diferenciação entre eles e no cultivar BRASMAX Bônus IPRO as maiores medias foram obtidas ao empregar $\mathrm{AB}$ e Trich como tratamentos no solo para a cultura da soja. Nas peneiras menores Ø5.5 e Ø5.0 o controle foi quem manifestou as maiores percentagens de grãos retidos nessas duas peneiras, o que mostra que os tratamentos com biológicos em relação ao controle promoveram nas cultivares avaliadas uma maior concentração de grãos nas peneiras maiores (Ø7.0, Ø6.5 e Ø6.0).

$\mathrm{Na}$ variável RP e PRGRP, verificou-se que o emprego de biológicos foi mais efetivo para se obter a maior percentagem de grãos retidos nas peneiras de maior peso se comparado com o controle (Quadro 2). Na variável RP todos os biológicos e sua combinação $(1+2+3)$ foram superiores ao controle para as cultivares BRASMAX Foco IPRO e BRASMAX Bônus IPRO, já na cultivar BRASMAX Desafio IPRO o controle não se diferenciou do tratamento $1+2+3$, o que indica que os biológicos promoveram uma maior percentagem de grãos nas principais peneiras avaliadas em relação ao controle, o que traz como resultado uma melhor qualidade fisiológica dessas sementes obtidas. Pádua et al. (2010) ao avaliar os efeitos do tamanho de semente sobre a qualidade fisiológica de três cultivares de soja, verificaram que as sementes classificadas em diferentes peneiras evidenciam diferentes qualidades fisiológicas, sendo as classificadas em peneira $7 \mathrm{~mm}$ as que manifestaram as melhores porcentagens de germinação e de vigor, colaborando com os resultados deste trabalho. Ao avaliar dois cultivares de soja e diferentes tamanhos de sementes, Dos Santos et al. (2005) encontraram que as características porcentagem e a velocidade de emergência das plântulas no campo foram afetadas com o armazenamento. Estes trabalhos mostram a importância de avaliar-se o tamanho dos grãos de soja e sua resposta na qualidade fisiológica (TRÉS et al., 2010; PICCININ et al. 2011; COELHO et al., 2019).

\section{Conclusão}

Os resultados obtidos mostram que o emprego de biológicos aplicados de modo post-emergente na cultura da soja não alteram a produtividade dos grãos, independentemente da cultivar. Mas, para a cultivar BRASMAX Bônus IPRO incrementou o rendimento de peneira e a massa de mil grãos. O emprego de biológicos aplicados de modo post-emergente na cultura da soja promove uma melhor qualidade fisiológica nas cultivares empregadas se comparadas ao controle, ao considerar os diâmetros de Ø6.0 e Ø6.5.

\section{Agradecimentos}

Agradecemos à UFMS - CPCS, por garantir o espaço físico para a realização deste trabalho. Ao produtor Evandro Loeff e sua equipe, pela transferência do material biológico usado no experimento. Agradecer o apoio financeiro do Coordenador de Aperfeiçoamento de Pessoal de Nível Superior - Brasil (CAPES) aos Professores Visitantes JGA e AMZ. À Fundação Chapadão, por permitir o uso de máquinas e sementes para a implementação do experimento de campo.

\section{Referências}

ALBERTO-CASAS, M. et al. Respuesta de soya (Glycine max (L) Merr) a la inoculación con Azospirillum y Bradyrhizobium. Cult. Trop., v.40, n.1, p.a02-e02, 2019.

AMK (2019) AMK orgânicos e biológicos: benefícios do uso do Bio-Ax. Disponível em: http://amkbio.com.br. Acesso em: 13 out. 2019.

ARMENDARIZ, A.L. et al. Impact of double inoculation with Bradyrhizobium japonicum E109 and Azospirillum brasilense Az39 on soybean plants grown under arsenic stress. Plant Physiol. Bioch., v. 138, p. 26-35, 2019. doi: 10.1016/j.plaphy.2019.02.018

BERNARDO, J.T. et al. Isolamento on farm de Trichoderma: uma ferramenta no controle de doenças de solo para os agricultores no Brasil. Rev. Elet. Cient. Uergs, v.5, p.263-270, 2019. doi: 10.21674/2448-0479.53.263-270

BETTIOL, W.; MORANDI, M.A.B. (Ed.). Biocontrole de doenças de plantas: uso e perspectivas. Jaguariúna: Embrapa Meio Ambiente, 2009.

BHERING, L.L. Rbio: a tool for biometric and statistical analysis using ther platform. Crop. Breed. Appl. Biot., v.17, p.187-190, 2017. doi: 10.1590/1984-70332017v17n2s29

BIRUEL, R.P.; PAULA, R.C.; AGUIAR, I.B. Germinação de sementes de Caesalpinia leiostachya (benth.) Ducke (pau-ferro) classificadas pelo tamanho e pela forma. Rev. Árvore, v. 34, n. 2, p. 197-204, 2010. doi: 10.1590/S0100-67622010000200001.

BRASIL. Ministério da Agricultura, Pecuária e Abastecimento. Regras para análise de sementes, Brasília: MAPA, 2009.

BRASMAX. Produtividade: A Brasmax tem rendimento. Disponível em: http://www.brasmaxgenetica.com.br/ . Acesso em: 13 out. 2019.

CARBONELL, S. A. M. et al. Tamanho de grão comercial em cultivares de feijoeiro. Ciênc. Rural, v.40, p.2067-2073, 2010. doi: $10.1590 / \mathrm{S} 0103-84782010005000159$

CASSÁN, F. et al. Azospirillum brasilense Az39 and Bradyrhizobium japonicum E109, inoculated singly or in combination, promote seed germination and early seedling growth in corn (Zea mays L.) and soybean (Glycine max L.). Eur. J. Soil Biol., v. 45, n. 1, p. 28-35, 2009. doi: 10.1016/j. ejsobi.2008.08.005

COELHO, E. B. et al. Influência do tamanho da semente na qualidade fisiológica da soja. Ipê Agron. J., v. 3, n. 1, p. 71-79, 2019.

CONAB (Companhia Nacional de Abastecimento). Acompanhamento da safra brasileira: grãos, Sexto levantamento [Internet] março 2019. Brasília: CONAB, 2019.

CORREIA, N. M.; DURIGAN, J. C. Culturas de cobertura e sua influência na fertilidade do solo sob sistema de plantio direto (SPD). Biosc. J., v. 24, n. 4, p. 20-31, 2008. 
DOS SANTOS, P. M. et al. Efeito da classificação por tamanho da semente de soja na sua qualidade fisiológica durante o armazenamento. Acta Sci. Agron., v. 27, n. 3, p. 395-402, 2005. doi: 10.4025 /actasciagron.v27i3.1398

FELISBERTO, G. et al. Agronomic performance of RR soybean cultivars using different pre-sowing desiccation periods and distinct post-emergence herbicides. Afr. J. Agric. Res., v. 10, n. 34, p. 3445-3452, 2015. doi: 10.5897/AJAR2015.9853

FRANCO, A. D. et al. Respuesta de la soya a inoculantes microbianos en el norte de Tamaulipas, México. Rev. Mexicana Cienc. Agric., v.6, n.2, p.227-238, 2015.

GÖRGEN, C. A. et al. Controle do mofo-branco com palhada e Trichoderma harzianum 1306 em soja. Pesq. Agropec. Bras., v. 44, n. 12, p. 1583-1590, 2009.

JUNGES, E. et al. Trichoderma spp. na Produção de Mudas de Espécies Florestais. Floresta e Ambiente, v. 23, n. 2, p. 237-244, 2016. doi: 10.1590/2179-8087.107614

KHAN, M.S. et al. Inoculation Effects of Associative Plant Growth-Promoting Rhizobacteria on the Performance of Legumes. In: ZAIDI A., KHAN M., MUSARRAT J. (eds) Microbes for Legume Improvement. Springer, Cham, 2017. p. 20-34.

MACHADO, D. F. M. et al. Trichoderma no Brasil: o fungo e o bioagente. Revista de Ciências Agrárias, v. 35, n. 1, p. 274-288, 2012.

MASTOURI, F., BJÖRKMAN, T.; HARMAN, G. E. Seed treatment with Trichoderma harzianum alleviates biotic, abiotic, and physiological stresses in germinating seeds and seedlings. Phytopathology, v. 100, n. 11, p. 1213-1221, 2010. doi: 10.1094/
PHYTO-03-10-0091.

PÁDUA, G. P. et al. Influência do tamanho da semente na qualidade fisiológica e na produtividade da cultura da soja. Rev. Bras. Sementes, v.32, n.3, p.9-16, 2010. doi: 10.1590/S010131222010000300001

PICCININ, G. G. et al. Relação entre o tamanho e a qualidade fisiológica e sanitária de sementes de soja. Agrarian, v.5, n.15, p.20-28, 2011.

POMMERESCHE, R.; HANSEN, S. Examining root nodule activity on legumes. Fertil Crop Technical Note, p.1-4, 2017.

REGO, C. H. Q. et al. Co-inoculation with Bradyrhizobium and Azospirillum Increases Yield and Quality of Soybean Seeds. Agron. J., v.110, n.6, p.1-7, 2018. doi: 10.2134/agronj2018.04.0278

SOARES, I. O. et al. Interaction between soybean cultivars and seed density. Am. J. Plant Sci., v. 6, n. 9, p. 1425-1434, 2015. doi: 10.4236/ajps.2015.69142

TRÉS, S. P. et al. Avaliação do vigor em sementes de soja (Glycine max L. Merrill) em função do tamanho. Cultivando o saber, v. 3, n. 2, p. 31-37, 2010.

USDA. United States Department of Agriculture. World Agricultural Supply and Demand Estimates, 2019.

VAL, B. H. P. et al. Diversidade genética de genótipos de soja por meio de caracteres agromorfológicos. Ciên. Tecnol. Fatec-JB, v. 6, n. 1, p. 72-83, 2014.

ZUFFO, A. M. et al. Co-inoculation of Bradyrhizobium japonicum and Azospirillum brasilense in the soybean crop. Rev Ciênc. Agrár., v. 38, n. 1, p. 87-93, 2015. 